\title{
An Extension of Maximal Covering Location Problem based on the Choquet Integral
}

\author{
Aleksandar Takači ${ }^{1}$, Ivana Štajner-Papuga ${ }^{2}$, Darko Drakulić ${ }^{3}$, \\ Miroslav Marić ${ }^{4}$
}

${ }^{1}$ Faculty of Technology, University of Novi Sad, Bulevar Cara Lazara 1, 21000

Novi Sad, Serbia; atakaci@uns.ac.rs

${ }^{2}$ Department of Mathematics and Informatics, Faculty of Sciences, University of

Novi Sad, Trg D. Obradovića 4, 21000 Novi Sad, Serbia; ivana.stajner-

papuga@dmi.uns.ac.rs

${ }^{3}$ Faculty of Philosophy, University of East Sarajevo, Alekse Šantića 1, 71420 Pale, Bosnia and Herzegovina; ddrakulic@ffuis.edu.ba

${ }^{4}$ Faculty of Mathematics, University of Belgrade, Studentski trg 16, 11000

Beograd, Serbia; maricm@ matf.bg.ac.rs

\begin{abstract}
The aim of this paper is to demonstrate the applicability of the Choquet integral, a well-known fuzzy integral, in the Maximal Covering Location Problem (MCLP). Possible benefits of the used integral, which is based on monotone set functions, include the flexibility of a monotone set function, which is in the core of the Choquet integral, for modeling the Decision Maker's behavior. Various mathematical models of the Maximal Covering Location Problem are given. The approach, based on the Choquet integral versus the standard approach, is thoroughly discussed and illustrated by several examples.
\end{abstract}

Keywords: Maximal Covering Location Problem; monotone set function; Choquet integral

\section{Introduction}

Many problems from the real world contain different uncertainties, ambiguities, and vagueness, so their mathematical models obtained with the classical mathematical techniques are not fully accurate. Fuzzy sets and different probabilistic methods are the most frequently used techniques for modeling problems from the real world. This study introduces a new method for modeling the Maximal Covering Location Problem (MCLP) by using a well-known fuzzy integral, the Choquet integral.

The Maximum Covering Location Problem (MCLP) was defined by Church and ReVelle in [5] and it represents a very important class of problems in operations research. They defined MCLP as follows: "Maximize the coverage within a 
desired service distance $S$ by locating a fixed number of facilities". In other words, the aim of MCLP is locating facilities on a given network in such a manner that they cover as many locations as possible. This class has a decisive role in many real world problems, such as locating shops, gas stations, bus stations, hospitals and other emergency services. Similar classes of problems include the Location Set Covering Problem (LSCP) and Minimal Covering Location Problem (MinCLP). The aim of LSCP is to cover all locations with as few facilities as possible, and the aim of MCLP is to cover as many locations as possible with a fixed number of facilities. In all models, the networks are represented by distances between locations (or travel times between them). Location coverage depends on the distance (or travel time) to the nearest facility and it depends on the given value called the coverage radius. In the classical case, those values are represented by real numbers, but in the "real-world" problems, those values are not fully determined, and they can contain different levels of vagueness, e.g., "the coverage radius is between 10 and 20 kilometers", "the travel time is around 20 minutes" or "it is pretty close". These linguistic ambiguities can be modeled by using different types of fuzzy numbers. Many authors developed different fuzzy MCLP models (FMCLP) and the most common approach is using fuzzy numbers for the radius of coverage. In the classical model of MCLP, each location is either covered or uncovered, while in FMCLP models, the locations could also be partially covered. The main question of FMCLP is how to treat partially covered locations. Depending on the nature of the problems, the degree of location coverage could be calculated using $t$-norms and $t$-conorms ([16]). This study takes into consideration another issue, namely the interaction between facilities which need to be optimally arranged. Now, the Choquet integral is being used in order to take into the account the different interactions between facilities which should yield a better quality solution.

This paper is organized as follows: in Section 2, a brief literature overview related to MCLP, FMCLP and the usage of fuzzy sets in location problems is presented. Section 3 includes certain basic mathematical notions, such as fuzzy sets, fuzzy numbers and the Choquet integral are given. Section 4 contains a new model of FMCLP based on the Choquet integral, while the last section offers some concluding remarks.

\section{Literature Overview}

As mentioned above, MCLP was developed by Church and ReVelle (1974) [5]. Different MCLP models were presented in the following years, like MCLP on the plane (Church, 1984 [6]), capacitated MCLP (Current and Storbeck, 1988 [7]), probabilistic MCLP (ReVelle and Hogan, 1989 [20]) and implicit MCLP (Murray et al. 2010 [17]). An exhaustive review of the covering problems and MCLP can be found in [12]. 
In recent years, several fuzzy models for the covering location problem have been presented. Darzentas in [8] presented a discrete location problem with fuzzy accessibility criteria and formulated it with an application of the set partitioning type of integer programming. Perez et al. in [19] presented that position of the facility in real applications can be full of linguistic vagueness, and they modeled them by using networks with fuzzy values. These fuzzy values appropriately describe the network nodes, lengths of paths, weight of nodes, etc. Batanovic et al. in [1] described the application of fuzzy sets in modeling the maximum covering location problems for networks in uncertain environments. They modeled distance (traveling times) from a facility site to demand nodes by fuzzy sets. Davari et al. in [9] presented a MCLP model with fuzzy variables for travel times for any pairs of nodes.

\section{Definitions and Preliminaries}

\subsection{Discrete Choquet Integral}

Since this discreteness is highly tangible in applications, the short overview of the discrete case, i.e., basic information on the discrete Choquet integral is given in this section.

It has to be emphasized that this type of integral is a highly applicable aggregation operator (see [11,14]). The Choquet integral generalizes the so-called additive operators, e.g., the OWA (the ordered weighted averaging operators, see [23]) and the weighted mean.

The first necessary notion is the one of a fuzzy measure. Firstly, let $X$ be a set of criteria, that is, let it be a set of all input values.

Definition 3.1 A set function $\mu: P(X) \rightarrow[0, \infty)$ is a fuzzy measure, if the following it satisfied

- $\mu(\varnothing)=0$,

- for arbitrary $A, B \in P(X)$, if $A \subset B$ then $\mu(A) \leq \mu(B)$ (monotonicity).

Now, the triplet $(X, P(X), \mu)$ is a fuzzy measure space $([2,3,13,22])$. In general, instead of $P(X)$ some $\sigma$-algebra of subsets of $X$ can be used.

As already mentioned, for the purpose of this research the focus is on a discrete case, i.e., on simple functions - functions that can assume only a finite number of values. Therefore, the following form of functions will be observed

$$
f: X \rightarrow\left\{\omega_{1}, \omega_{2}, \ldots \omega_{n}\right\}
$$

where $\omega_{i} \in[0, \infty)$ and the working assumption, with no influence on generality, is $0 \leq \omega_{1}<\omega_{2}<\cdots<\omega_{n} \leq 1$. 
Moreover, based on the type of problems that will be investigated in the future, it is sufficient to observe simple functions with values in $[0,1]$ and normalized fuzzy-measures, i.e., further it will be assumed that $\left\{\omega_{1}, \omega_{2}, \ldots \omega_{n}\right\} \subseteq[0,1]$ and $\mu: P(X) \rightarrow[0, \infty)$ is a fuzzy measure.

The definition of the Choquet integral for the discrete case follows ([4]).

Definition 3.2 The Choquet integral of an arbitrary simple function $f: X \rightarrow$ $\left\{\omega_{1}, \omega_{2}, \ldots \omega_{n}\right\}$, based on a fuzzy measure has the following form

$$
\text { (C) } \int_{X} f \mathrm{~d} \mu=\sum_{i=1}^{n}\left(\omega_{i}-\omega_{i-1}\right) \cdot \mu\left(\Omega_{i}\right),
$$

where $\Omega_{i}=\left\{x \mid f(x) \geq \omega_{i}\right\}$ and $\omega_{0}=0$ and $\mu$ is a fuzzy measure.

More on the Choquet integral can be found in [2, 3, 4, 10, 15, 18], to just name a few sources.

In general, the universality of fuzzy integrals as aggregation operators is deducted from the minimal restrictions imposed on set function that is in its core. The fact that the Choquet integral, discussed in this paper, covers many well-known classical aggregation operators can be illustrated by the following example (see [11]).

\section{Example 3.1}

- $\quad$ For the fuzzy measure $\mu: P(X) \rightarrow[0,1]$, given by

$$
\mu(X)=1 \text { and } \mu(A)=0 \text { for } A \neq X,
$$

the corresponding Choquet integral coincides with the classical minimum.

- $\quad$ For the fuzzy measure $\mu: P(X) \rightarrow[0,1]$, given by

$$
\mu(\varnothing)=0 \text { and } \mu(A)=1 \text { for } A \neq \emptyset \text {, }
$$

the corresponding Choquet integral coincides with the classical maximum.

- $\quad$ For the fuzzy measure $\mu: P(X) \rightarrow[0,1]$, given by

$$
\mu(A)=0 \text { for } \operatorname{card}(A) \leq n-k \text { and } \mu(A)=1 \text { otherwise, }
$$

the corresponding Choquet integral coincides with the classical $k$-order statistic.

- $\quad$ For the fuzzy measure $\mu: P(X) \rightarrow[0,1]$, given by

$$
\mu(A)=\frac{\operatorname{card}(A)}{\operatorname{card}(X)},
$$

the corresponding Choquet integral coincides with the classical arithmetic mean.

- $\quad$ For the fuzzy measure $\mu: P(X) \rightarrow[0,1]$, given by

$$
\mu(A)=\sum_{j=0}^{\operatorname{card}(A)-1} w_{n-j},
$$

where $w_{i}$ are pre-given weights, the corresponding Choquet integral coincides with the OWA operator. 
The main drawback for the practical use of the Choquet integral is the number of sets that need a predefined value of the fuzzy measure. If the observed function has a range of cardinality $n$, a Decision Maker needs to predefine $2^{n}$ values. One of the possible ways for simplifying a Decision Maker's task is to define values only for singletons and to aggregate the remaining values by some aggregating operator. Since monotonicity of measure is essential for this integral, this can be done by a t-conorm, i.e., if the fuzzy measure $\mu$ is the so-called $S$-decomposable measure.

Definition 3.3 [18] A set function $\mu: P(X) \rightarrow[0,1]$ that satisfies the following

- $\mu(\varnothing)=0$,

- $\mu(A \cup B)=S(\mu(A), \mu(B))$ for $A \cap B=\emptyset$,

where $S$ is a t-conorm, is called the $S$-decomposable measure.

A t-conorm is a binary operation $S:[0,1]^{2} \rightarrow[0,1]$, that is commutative, nondecreasing, associative and has zero as the neutral element. Elementary examples of continuous t-conorms are:

- $S_{\mathrm{M}}(x, y)=\max (x, y)-$ maximal,

- $S_{\mathrm{P}}(x, y)=x+y-x y-$ probabilistic,

- $S_{\mathrm{L}}(x, y)=\min (x+y, 1)-$ Lukasiewicz.

where $x, y \in[0,1]$. More on t-conorms and t-norms (dual operations) can be found in $[16,18]$, among others. Also, the sources $[11,14]$ offer more general background on aggregation operators.

Since t-conorms are associative operations, they can easily be extended to $n$-ary operators and used for calculating measures of non-singleton sets. Forms of $n$-ary operators for three previously mentioned basic t-conorms are given by the following example.

Example 3.2 Let $\left\{x_{1}, x_{2}, \ldots, x_{k}\right\}$ be an arbitrary subset of $X$. If $\mu$ is a $S$ decomposable measure, and values $\mu\left(\left\{x_{i}\right\}\right)$ are predefined, then the value $\mu\left(\left\{x_{1}, x_{2}, \ldots, x_{k}\right\}\right)$ can be calculated as follows (see [16])

- $\quad$ if $S=S_{\mathrm{M}}$

- $\quad$ if $S=S_{\mathrm{P}}$

$$
\mu\left(\left\{x_{1}, x_{2}, \ldots, x_{k}\right\}\right)=\max \left(\mu\left(\left\{x_{1}\right\}\right), \ldots, \mu\left(\left\{x_{k}\right\}\right)\right),
$$

$$
\mu\left(\left\{x_{1}, x_{2}, \ldots, x_{k}\right\}\right)=1-\prod_{i=1}^{k}\left(1-\mu\left(\left\{x_{i}\right\}\right)\right),
$$

- $\quad$ if $S=S_{\mathrm{L}}$

$$
\mu\left(\left\{x_{1}, x_{2}, \ldots, x_{k}\right\}\right)=\min \left(\sum_{i=1}^{k} \mu\left(\left\{x_{i}\right\}\right), 1\right) .
$$

Due to the nature of the problem that will be investigated further on, the focus of this paper is on the discrete case, i.e., when the observed set of input values is finite $X=\left\{x_{1}, x_{2}, \ldots, x_{n}\right\}$. 


\subsection{MCLP - Classical Case}

As mentioned in Section 1, MCLP was introduced by Church and ReVelle in 1974 [5], with the following mathematical model:

$$
\begin{array}{ll}
\text { maximize } & g=\sum_{i \in I} a_{i} y_{i} \\
\text { subject to } & \sum_{j \in N_{i}} x_{j} \geq y_{i}, \forall i \in I \\
& \sum_{j \in J} x_{j}=P \\
& x_{j} \in\{0,1\}, \forall j \in J \\
& y_{i} \in\{0,1\}, \forall i \in I
\end{array}
$$

where

$I$ - set of locations (indexed by $i$ )

$J-$ set of eligible facility sites (indexed by $j$ )

$S$ - radius of coverage

$d_{i j}$ - travel time from location $i$ to location $j$

$x_{j}=\left\{\begin{array}{l}1, \text { if facility is located at location } j \\ 0, \text { otherwise }\end{array}\right.$

$a_{i}$ - population in node $i$

$P$ - number of facilities

$N_{i}=\left\{j \mid d_{i j} \leq S\right\}-$ set of all facilities $j$ which cover location $i$

In this paper, population in a node $a_{i}$ is not considered, but it does not reduce the generality of the problem.

$N_{i}$ is the set of facility sites and it provides location coverage, i.e., location is covered if the distance between it and some facility is less than the predefined radius $S$, and location is not covered otherwise. A demand node is "covered" when the closest facility to that node is at a distance less than or equal to $S$. A demand node is "uncovered" when the closest facility to that node is at a distance greater than $S$. The objective is to maximize the number of people served or "covered" within the desired service distance. Constraints of the type (1) allow $y_{i}$ to equal 1 only when one or more facilities are established at sites in the set $N_{i}$ (that is, one or more facilities are located within the $S$ distance units of the demand point $i$ ). The number of facilities allocated is restricted to equal $P$ in constraint (2). The solution to this problem specifies not only the largest amount of population that can be covered, but the $P$ facilities that achieve this maximal coverage.

This condition is modeled by classical logic and each location could be fully covered or uncovered, and that fact gives motivation for the introduction of fuzzy numbers in modeling MCLP. 


\subsection{MCLP via Fuzzy Numbers (FMCLP)}

The main idea of using fuzzy numbers in modeling MCLP is the introduction of vagueness in location covering. FMCLP is the extension of MCLP, where some conditions are represented with fuzzy numbers and in FMCLP, the location can be covered, uncovered or partially covered ([21]). Depending on the nature of the problem, different aggregation operators (max, arithmetic average, median, min...) can be used to calculate the degree of partial coverage of a location. In the following model of FMCLP, max operator is used, but other operators can be used in a similar way.

$$
\begin{array}{ll}
\text { Maximize } & g=\sum_{i \in I} y_{i} \\
\text { subject to } & \max x_{j} \cdot c_{i j} \geq y_{i}, \forall i \in I \\
& \sum_{j \in J} x_{j}=P \\
& x_{j} \in\{0,1\}, \forall j \in J \\
& y_{i} \in[0,1], \forall i \in I
\end{array}
$$

where

$I$ - set of locations (indexed by $i$ )

$J$ - set of eligible facility sites (indexed by $j$ )

$S$ - radius of complete coverage

$s$ - fuzzy radius of partial coverage

$d_{i j}$ - travel time from location $i$ to location $j$

$x_{j}=\left\{\begin{array}{l}1, \text { if facility is located at location } j \\ 0, \text { otherwise }\end{array}\right.$

$P$ - number of facilities

$c_{i j}=\left\{\begin{array}{l}1, d_{i j} \leq S \\ 0, d_{i j} \leq S+s \quad \text { - matrix of coverage } \\ e \in(0,1), \text { otherwise }\end{array}\right.$

The main difference between MCLP and FMCLP lies in the coverage radius. In the presented FMCLP model, the coverage radius is a fuzzy number (rightshoulder fuzzy number) which allows partial coverage. Now, the coverage degree $y_{i}$ is a number in the unit interval and the coverage matrix determines its value. The exact value of $y_{i}$ is defined by a membership function and depends on the nature of the problem.

Travel time could also be a fuzzy number (these are usually triangular fuzzy numbers) and that modification results in another FMCLP model. In that model, partial coverage is defined by the intersection of the fuzzy radius (represented by a 
right-shoulder fuzzy number) and fuzzy travel time (represented by a triangular fuzzy number). More on this approach and its applications in other location problems can be found in [21].

\section{Fuzzy Integral-based Models of Fuzzy Maximal Covering Location Problem}

The main motivation for proposing new models is taking into consideration the interaction measure between facilities. In all the existing models, the facilities could not interact with each other and each facility has the same importance. Thus, the level of interaction, or the level of joint importance, is given by a monotone set-function. Together with the usage of the Choquet integral, it forms a new, promising powerful extended model of FMCLP.

The basics of the proposed model are

- $\quad P$ - number of facilities [integer],

- $X=\left\{L_{1}, L_{2}, \ldots, L_{R}\right\}-$ set of all locations,

- $Y=\left\{Y_{1}, Y_{2}, \ldots, Y_{P}\right\}-$ set of all facilities,

- $\mu: \mathrm{P}(X) \rightarrow[0,1]-$ measure of interaction for different facilities modeled by a monotone set function,

- $\omega_{i, j} \in[0,1]$ - degree of coverage for location $L_{i}$ by the $j$-th facility,

- $A$ is the intended layout of facilities from $Y$ over the location set $X$.

The following constitute the proposed model:

MODEL Ch - the Choquet based model

$$
\begin{gathered}
f_{L_{i}}: Y \rightarrow\left\{\omega_{i, 1}, \omega_{i, 2}, \ldots, \omega_{i, m}\right\}, \quad i=\{1, \ldots, R\}, \\
g(A)=\sum_{i}(C) \int f_{L_{i}} \mathrm{~d} \mu .
\end{gathered}
$$

Namely, the functions (1) give the degree of coverage of each node by the facilities from $Y$, while formula (2) is the function whose maxima, for different positions of the facilities from $Y$, is needed. Given this, the layout of the facilities from $Y$ for which (2) is maximal is the optimal layout. The monotone set function $\mu$ is predefined by a Decision Maker and can be interpreted as a quality measure of facilities and their interaction. The optimal case is obtained when the Decision Maker is able to provide the values of $\mu$ for all subsets of $Y$. By doing that, the Decision Maker expresses their own opinion on how the facilities in question interact, i.e., how "strong" they are together. However, this means that the Decision Maker should single-handedly provide $2^{P}$ values, which would be an unreasonable request. An acceptable solution is to ask for values only for 
singletons, and to use an aggregation operator, e.g., a t-conorm, acceptable for the Decision Maker's behavior. The following algorithm is proposed

- $\quad$ STEP I: Acquiring values for $\mu\left(\left\{Y_{1}\right\}\right), \mu\left(\left\{Y_{2}\right\}\right), \ldots, \mu\left(\left\{Y_{P}\right\}\right)$,

- STEP II: Selection of the appropriate t-conorm:

- $\quad S_{\mathrm{M}}$ - if the strongest facility dominates all others,

- $\quad S_{\mathrm{P}}$ - if facilities complement each other, with overlaps,

- $\quad S_{L}$ - if facilities complement each other, with negligible overlaps,

- $\quad$ STEP III: Calculation of values for

$$
\mu\left(\left\{Y_{j_{1}}, Y_{j_{2}}, \ldots, Y_{j_{k}}\right\}\right),\left\{j_{1}, j_{2}, \ldots, j_{k}\right\} \subseteq\{1,2, \ldots, P\},
$$

by formulas from Example 3.2.

Remark 4.1 Step II offers only three options because they can easily be interpreted by real life concepts such as domination (one facility is much more important to the Decision Maker and its influence is strong enough to overcome influences of other facilities) and negligible overlaps (influences of different facilities can be directed to the same area, however they do not compete with each other). Of course, the set of t-conorms is much wider (see [16]) and some other $t$ conorms can be chosen depending on the decision maker's preferences.

The behavior of the proposed model depending on the Decision Maker's personal perceptions of quality and interaction of facilities is illustrated by the following propositions.

Proposition 4.1 Let $X=\left\{L_{1}, L_{2}, \ldots, L_{R}\right\}$ be the set of all locations, $Y=$ $\left\{Y_{1}, Y_{2}, \ldots, Y_{P}\right\}$ the set of all facilities, $\omega_{i, j} \in[0,1]$ degree of coverage for location $L_{i}$ by the $j$-th facility and let $A$ be the intended layout of facilities from $Y$ over the location set $X$.

If qualities of facilities $Y=\left\{Y_{1}, Y_{2}, \ldots, Y_{P}\right\}$ are estimated by two different decision makers, i.e., if two $\mathrm{S}$ - decomposable measures $\mu_{1}: \mathrm{P}(Y) \rightarrow[0,1]$ and $\mu_{2}: \mathrm{P}(Y) \rightarrow$ $[0,1]$ based on the same t-conorm $\mathrm{S}$ are assigned, such that

$$
\mu_{1}\left(\left\{Y_{j}\right\}\right) \leq \mu_{2}\left(\left\{Y_{j}\right\}\right)
$$

for all $j \in\{1,2, \ldots, P\}$, then the following holds

$$
g_{\mu_{1}}(A) \leq g_{\mu_{2}}(A) .
$$

Proof. Since $\mu_{1}: \mathrm{P}(Y) \rightarrow[0,1]$ and $\mu_{2}: \mathrm{P}(Y) \rightarrow[0,1]$ are $S$-decomposable measures and since for all singletons $\left\{Y_{j}\right\}, j \in\{1,2, \ldots, P\}$, holds $\mu_{1}\left(\left\{Y_{j}\right\}\right) \leq$ $\mu_{2}\left(\left\{Y_{j}\right\}\right)$, based on monotonicity of t-conorms (see [16]), it follows that $\mu_{1}(E) \leq$ $\mu_{2}(E)$ for all $E \in \mathrm{P}(Y)$. Now, based on properties of the Choquet integral (see $[2,3])$, it holds

$$
\text { (C) } \int f_{L_{i}} \mathrm{~d} \mu_{1} \leq(C) \int f_{L_{i}} \mathrm{~d} \mu_{2},
$$

for all corresponding functions $f_{L_{i}}: Y \rightarrow\left\{\omega_{i, 1}, \omega_{i, 2}, \ldots, \omega_{i, m}\right\}, \quad i \in\{1,2, \ldots, R\}$. Therefore, the claim holds. 
Proposition 4.2 Let $X=\left\{L_{1}, L_{2}, \ldots, L_{R}\right\}$ be the set of all locations, $Y=$ $\left\{Y_{1}, Y_{2}, \ldots, Y_{P}\right\}$ the set of all facilities, $\omega_{i, j} \in[0,1]$ degree of coverage for location $L_{i}$ by the $j$-th facility and let $A$ be the intended layout of facilities from $Y$ over the location set $X$.

If interactions of facilities $Y=\left\{Y_{1}, Y_{2}, \ldots, Y_{P}\right\}$ are estimated by two different decision makers such that two different $S$-decomposable measures, $S_{1}$ decomposable measure $\mu_{1}: \mathrm{P}(Y) \rightarrow[0,1]$ and $S_{2}$-decomposable measure $\mu_{2}: \mathrm{P}(Y) \rightarrow[0,1]$, are assigned in the following manner

$$
\mu_{1}\left(\left\{Y_{j}\right\}\right)=\mu_{2}\left(\left\{Y_{j}\right\}\right),
$$

for all $j \in\{1,2, \ldots, P\}$, and $S_{1} \leq S_{2}$, then the following holds

$$
g_{\mu_{1}}(A) \leq g_{\mu_{2}}(A) \text {. }
$$

Proof. Measures $\mu_{1}: \mathrm{P}(Y) \rightarrow[0,1]$ and $\mu_{2}: \mathrm{P}(Y) \rightarrow[0,1]$ are S-decomposable measures, therefore, based on the starting assumption $S_{1} \leq S_{2}\left(S_{1}(x, y) \leq\right.$ $S_{2}(x, y)$ for all $x, y \in[0,1]$, see [16]), it holds $\mu_{1}(E) \leq \mu_{2}(E)$ for all $E \in \mathrm{P}(Y)$. Now, due to properties of the Choquet integral (see [2,3]), analogous to the proof of the previous proposition, the claim holds.

Remark 4.2 Since for three proposed t-conorms holds $S_{M} \leq S_{P} \leq S_{L}$, it is obvious that for the resulting mark for a certain layout $A$ holds $g_{\mu_{S_{M}}}(A) \leq$ $g_{\mu_{S_{P}}}(A) \leq g_{\mu_{S_{L}}}(A)$. That is, if facilities complement each other, instead having one that is dominant, the resulting mark is higher.

Additionally, although at first glance the introduction of $\mu$ seems to increase the computational complexity, this can be avoided, because in the implementations only few subsets are connected to a single node.

Proposition 4.3 The algorithm for calculation of the function $g(A)=$ $\sum_{i}(C) \int f_{L_{i}} d \mu$ has the maximal complexity of $O(R P \log P)$, where $P$ is the number of the given facilities and $R$ is the number of the observed locations.

Proof. The worst case, i.e., the maximal complexity, is reached when each location has a different deegree of coverage for all available facilities, that is when the range of function $f_{L_{i}}$ has exactly $P$ different elements, for all $i=\{1, \ldots, R\}$. In that case, $\int f_{L_{i}} \mathrm{~d} \mu=\sum_{k=1}^{P}\left(\omega_{i, k}-\omega_{i, k-1}\right) \cdot \mu\left(\Omega_{i, k}\right)$ has $P$ summands. Before calculation of this sum, it is necessary to sort elements from $\left\{\omega_{i, 1}, \omega_{i, 2}, \ldots, \omega_{i, P}\right\}$, i.e., to sort the set of all deegrees of coverage. This can be done in $O(P \log P)$ steps (by using, for example, Merge Sort). With sorted elements, computational complexity of this sum depends on the complexity of computing measures $\mu\left(\Omega_{i, 1}\right), \mu\left(\Omega_{i, 2}\right), \ldots, \mu\left(\Omega_{i, P}\right)$. From the definition of the $S$-measure $\mu$ and properties of t-conorms in general, follows that 


$$
\begin{gathered}
\mu\left(\Omega_{i, j}\right)=\mu\left(\left\{Y_{j}, Y_{j+1}, \ldots, Y_{P}\right\}\right)=S\left(\mu\left(\left\{Y_{j}\right\}\right), \mu\left(\left\{Y_{j+1}, \ldots, Y_{P}\right\}\right)\right) \\
=S\left(\mu\left(\left\{Y_{j}\right\}\right), \mu\left(\Omega_{i, j+1}\right)\right)
\end{gathered}
$$

which insures that integral $(C) \int f_{L_{i}} \mathrm{~d} \mu$ (with sorted elements of $\left.\left\{\omega_{i, 1}, \omega_{i, 2}, \ldots, \omega_{i, P}\right\}\right)$ can be computed with coplexity $O(P)$. Since there are $R$ summands in the function $g(A)$, the total complexity is $O(R P \log P)$.

Remark 4.3 In order to simplify the computational complexity and bring this concept closer to the Decision Maker, functions (1) can have linguistic values, i.e.,

$$
f_{L_{i}}: Y \rightarrow\{\text { none, poor, fair, good, full }\} .
$$

If $f_{L_{i}}\left(Y_{j}\right)=$ none, then node (location) $L_{i}$ is not in range of the facility $Y_{j}$ for the observed layout, etc. Of course, later on, linguistic values can be appropriately coded. In this case, the exact values of elements in sets $\left\{\omega_{i, 1}, \omega_{i, 2}, \ldots, \omega_{i, P}\right\}$ are known in advance and sorting can be done in $O(P)$ steps (by using, for example, Counting sort). Therefore, the total complexity is $O(P R)$.

\subsection{Examples}

The proposed model can be illustrated by the following simple setting. Let the assumption be that there are 6 locations with distances as in Figure 1 and two facilities to be located. Now, the set of locations is $X=\left\{L_{1}, \ldots, L_{6}\right\}$ and set of facilities is $Y=\left\{Y_{a}, Y_{b}\right\}$. However, since facilities will be positioned on certain locations, the notation will be $Y=\left\{Y_{a j}, Y_{b k}\right\}$ where $\{j, k\} \subset\{1,2, \ldots, 6\}$ depends on the intended position of a facility.

Let the assumption be that two layouts are under the consideration:

$$
\begin{aligned}
& \text { A: } Y=\left\{Y_{a 1}, Y_{b 6}\right\}, \\
& \text { B: } Y=\left\{Y_{a 2}, Y_{b 5}\right\},
\end{aligned}
$$

i.e., facilities $Y_{a}$ and $Y_{b}$ are located on locations $L_{1}$ and $L_{6}$, and $L_{2}$ and $L_{5}$, respectively. The first calculation is the implementation of the classical case, the second one is done via fuzzy numbers, while the third one is based on the model proposed in this paper. Since the quality (or influence) of facilities in question is the same for the first two approaches (given by examples 4.1 and 4.2), for the sake of simplicity, the following notations will be used:

$$
\begin{aligned}
& \text { A: } Y=\left\{Y_{1}, Y_{6}\right\}, \\
& \text { B: } Y=\left\{Y_{2}, Y_{5}\right\},
\end{aligned}
$$

which is the standard in MCLP problems. However, for the third approach, the quality of facility is relevant and this more complex notation will be used. 
Example 4.1 First, a classical MCLP problem without any fuzzy coverage will be used. Let it be supposed that the coverage radius is $5 \mathrm{~km}$, i.e., the function is defined in the following way

$$
f_{L_{i}}\left(L_{j}\right)=\left\{\begin{array}{c}
1, \text { if } \mathrm{d}\left(L_{i}, L_{j}\right) \leq 5 \mathrm{~km}, \\
0, \text { otherwise }
\end{array}\right.
$$

If the facilities are located in $L_{1}$ and $L_{6}$ then all six locations are covered. On the other hand, if the facilities are located in $L_{2}$ and $L_{5}$, only four locations are covered, the locations $L_{3}$ and $L_{6}$ are not covered by this solution. Therefore, the optimal solution is option A.

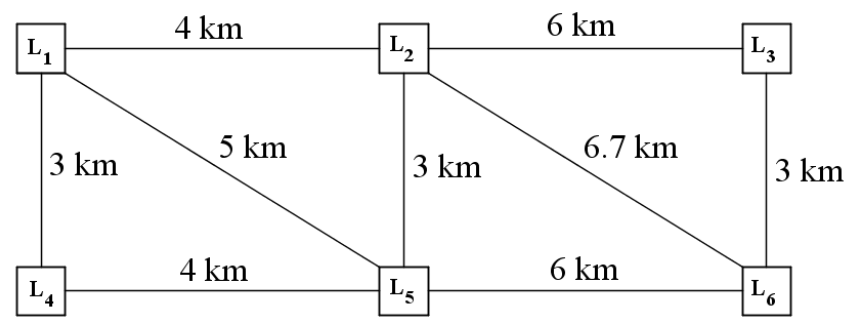

Figure 1

Location setting

Example 4.2 It will now be supposed that the location can be partially covered, i.e., FMCLP will be considered. The coverage radius for this approach is defined by the following function (see [21])

$$
f_{L_{i}}\left(L_{j}\right)=\left\{\begin{array}{cc}
1, & \text { if } \mathrm{d}\left(L_{i}, L_{j}\right) \leq 3 \mathrm{~km}, \\
-\frac{1}{4} \mathrm{~d}\left(L_{i}, L_{j}\right)+\frac{7}{4}, & 3 \mathrm{~km}<\mathrm{d}\left(L_{i}, L_{j}\right) \leq 7 \mathrm{~km}, \\
0, & \text { otherwise. }
\end{array}\right.
$$

For option A, the facilities are located in $L_{1}$ and $L_{6}$ and they are marked as $Y_{1}$ and $Y_{6}$ and

- $f_{L_{1}}:\left\{Y_{1}, Y_{6}\right\} \rightarrow\left\{\omega_{1,1}, \omega_{1,2}\right\}, \quad f_{L_{1}}\left(Y_{1}\right)=1, \quad f_{L_{1}}\left(Y_{6}\right)=0$;

- $f_{L_{2}}:\left\{Y_{1}, Y_{6}\right\} \rightarrow\left\{\omega_{2,1}, \omega_{2,2}\right\}, \quad f_{L_{2}}\left(Y_{1}\right)=0.75, f_{L_{2}}\left(Y_{6}\right)=0.075$;

- $f_{L_{3}}:\left\{Y_{1}, Y_{6}\right\} \rightarrow\left\{\omega_{3,1}, \omega_{3,2}\right\}, \quad f_{L_{3}}\left(Y_{1}\right)=0, \quad f_{L_{3}}\left(Y_{6}\right)=1$;

- $f_{L_{4}}:\left\{Y_{1}, Y_{6}\right\} \rightarrow\left\{\omega_{4,1}, \omega_{4,2}\right\}, \quad f_{L_{4}}\left(Y_{1}\right)=1, \quad f_{L_{4}}\left(Y_{6}\right)=0$;

- $f_{L_{5}}:\left\{Y_{1}, Y_{6}\right\} \rightarrow\left\{\omega_{5,1}, \omega_{5,2}\right\}, \quad f_{L_{5}}\left(Y_{1}\right)=0.5, \quad f_{L_{5}}\left(Y_{6}\right)=0.25$;

- $f_{L_{6}}:\left\{Y_{1}, Y_{6}\right\} \rightarrow\left\{\omega_{6,1}, \omega_{6,2}\right\}, \quad f_{L_{6}}\left(Y_{1}\right)=0, \quad f_{L_{6}}\left(Y_{6}\right)=1$.

Therefore, the coverage of location $L_{1}$ is $\max (1,0)=1$, for $L_{2}$ is $\max (0.75,0.075)=0.75, L_{3}$ is $1, L_{4}$ is $1, L_{5}$ is 0.5 and $L_{6}$ is 1 . Now, the coverage degree of the option $A$ is

$$
g(A)=1+0.75+1+1+0.5+1=5.25 .
$$


For layout B the following holds

- $f_{L_{1}}:\left\{Y_{2}, Y_{5}\right\} \rightarrow\left\{\omega_{1,1}, \omega_{1,2}\right\}$, $f_{L_{1}}\left(Y_{2}\right)=0.75, \quad f_{L_{1}}\left(Y_{5}\right)=0.5$;

- $f_{L_{2}}:\left\{Y_{2}, Y_{5}\right\} \rightarrow\left\{\omega_{2,1}, \omega_{2,2}\right\}$, $f_{L_{2}}\left(Y_{2}\right)=1, \quad f_{L_{2}}\left(Y_{5}\right)=1$;

- $f_{L_{3}}:\left\{Y_{2}, Y_{5}\right\} \rightarrow\left\{\omega_{3,1}, \omega_{3,2}\right\}$, $f_{L_{3}}\left(Y_{2}\right)=0.25, f_{L_{3}}\left(Y_{5}\right)=0.075$;

- $f_{L_{4}}:\left\{Y_{2}, Y_{5}\right\} \rightarrow\left\{\omega_{4,1}, \omega_{4,2}\right\}$, $f_{L_{4}}\left(Y_{2}\right)=0.5, \quad f_{L_{4}}\left(Y_{5}\right)=0.75$;

- $f_{L_{5}}:\left\{Y_{2}, Y_{5}\right\} \rightarrow\left\{\omega_{5,1}, \omega_{5,2}\right\}$, $f_{L_{5}}\left(Y_{2}\right)=1, \quad f_{L_{5}}\left(Y_{5}\right)=1$;

- $f_{L_{6}}:\left\{Y_{2}, Y_{5}\right\} \rightarrow\left\{\omega_{6,1}, \omega_{6,2}\right\}$, $f_{L_{6}}\left(Y_{2}\right)=0.075, f_{L_{6}}\left(Y_{5}\right)=0.25$.

and

$$
g(B)=0.75+1+0.25+0.75+1+0.25=4 .
$$

Again, layout A is optimal.

The following example illustrates the proposed model based on the Choquet integral. In this case, the quality of facilities, at least the Decision Maker's perception of that quality, influences the result.

Example 4.3 Let one consider option A. The values that describe the quality of each facility are $\mu\left(\left\{Y_{a 1}\right\}\right)$ and $\mu\left(\left\{Y_{b 6}\right\}\right)$, and they are provided by a Decision Maker. Their joint quality, i.e., the measure of how much they complement each other is $\mu\left(\left\{Y_{a 1}, Y_{b 6}\right\}\right)$, can be obtained, as presented in Example 3.1. It is assumed that the measure of an empty set is zero.

The next step is the calculation of the Choquet integral for each function according to the measure $\mu$. That is "coverage of the location $L_{i}$ ":

- $\quad(C) \int f_{L_{1}} \mathrm{~d} \mu=(1-0) \cdot \mu\left(\left\{y \mid f_{L_{1}} \geq 1\right\}\right)=\mu\left(\left\{Y_{a 1}\right\}\right)$,

- $\quad(C) \int f_{L_{2}} \mathrm{~d} \mu=(0.075-0) \cdot \mu\left(\left\{y \mid f_{L_{2}} \geq 0.075\right\}\right)+(0.75-0.075)$.

$\mu\left(\left\{y \mid f_{L_{2}} \geq 0.08\right\}\right)=0.075 \mu\left(\left\{Y_{a 1}, Y_{b 6}\right\}\right)+0.675 \mu\left(\left\{Y_{a 1}\right\}\right)$,

- $\quad(C) \int f_{L_{3}} \mathrm{~d} \mu=(1-0) \cdot \mu\left(\left\{y \mid f_{L_{3}} \geq 1\right\}\right)=\mu\left(\left\{Y_{b 6}\right\}\right)$,

- $\quad(C) \int f_{L_{4}} \mathrm{~d} \mu=(1-0) \cdot \mu\left(\left\{y \mid f_{L_{4}} \geq 1\right\}\right)=\mu\left(\left\{Y_{a 1}\right\}\right)$,

- $\quad(C) \int f_{L_{5}} \mathrm{~d} \mu=(0.25-0) \cdot \mu\left(\left\{y \mid f_{L_{5}} \geq 0.25\right\}\right)+(0.5-0.25)$. $\mu\left(\left\{y \mid f_{L_{5}} \geq 0.5\right\}\right)=0.25 \mu\left(\left\{Y_{a 1}, Y_{b 6}\right\}\right)+0.25 \mu\left(\left\{Y_{a 1}\right\}\right)$,

- $\quad(C) \int f_{L_{6}} \mathrm{~d} \mu=(1-0) \cdot \mu\left(\left\{y \mid f_{L_{6}} \geq 1\right\}\right)=\mu\left(\left\{Y_{b 6}\right\}\right)$.

The coverage degree of the $L_{1}-L_{6}$ layout is given by

$$
\begin{gathered}
g(A)=\sum_{i}(C) \int f_{L_{i}} \mathrm{~d} \mu= \\
2.925 \mu\left(\left\{Y_{a 1}\right\}\right)+2 \mu\left(\left\{Y_{b 6}\right\}\right)+0.325 \mu\left(\left\{Y_{a 1}, Y_{b 6}\right\}\right) .
\end{gathered}
$$

Similarly, for layout $L_{2}-L_{5}$, i.e., for option $B$, the coverage degree is

$$
\begin{gathered}
g(B)=\sum_{i}(C) \int f_{L_{i}} \mathrm{~d} \mu \\
=0.425\left(\mu\left(\left\{Y_{a 2}\right\}\right)+\mu\left(\left\{Y_{b 5}\right\}\right)\right)+3.15 \mu\left(\left\{Y_{a 2}, Y_{b 5}\right\}\right) .
\end{gathered}
$$


Let it be assumed that the qualities of two facilities in question are graded with, e.g., 0.6 and 0.8 and if overlaps are negligible, the $S_{L}$ can be used as the aggregation operator. For option A, if the facility of quality 0.6 is placed on location $L_{1}$, the following holds

$$
\mu\left(\left\{Y_{a 1}\right\}\right)=0.6, \mu\left(\left\{Y_{b 6}\right\}\right)=0.8, \mu\left(\left\{Y_{a 1}, Y_{b 6}\right\}\right)=1 \text { and } g(A)=3.68 \text {. }
$$

On the other hand, for option B, if the facility of quality 0.6 is placed on location $L_{2}$, the following holds

$$
\mu\left(\left\{Y_{a 2}\right\}\right)=0.6, \mu\left(\left\{Y_{b 5}\right\}\right)=0.8, \mu\left(\left\{Y_{a 2}, Y_{b 5}\right\}\right)=1 \text { and } g(B)=3.745 \text {. }
$$

Now, since the quality of facilities is taken into account, the result is different and the optimal solution is layout $B$.

While in MCLP and FMCLP the quality of facilities is not taken in to consideration, it has a high influence on the result in the proposed model. The flexibility of the proposed model can be additionally illustrated by the following example, that is the continuation of the previous one.

Example 4.4 If the positions of facilities in option A are inverted, i.e., if the layout is A: $Y=\left\{Y_{b 1}, Y_{a 6}\right\}$, the following holds

$$
\mu\left(\left\{Y_{b 1}\right\}\right)=0.8, \mu\left(\left\{Y_{a 6}\right\}\right)=0.6, \mu\left(\left\{Y_{b 1}, Y_{a 6}\right\}\right)=1 \text { and } g(A)=3.865 \text {. }
$$

That is, now this layout is better than layout $B$.

Remark 4.4 If the assumption is that all facilities are of the same quality, e.g., quality 1, the proposed model coincides with FMCLP.

As seen from the previous examples, the new model allows the quality of facilities, given by the measure $\mu$, to influence the final decision. All four examples are summarized in Table 1 . The optimal option is marked with *.

Table 1

Comparison of coverage degrees

\begin{tabular}{|c|c|c|c|c|}
\hline & MCLP & FMCLP & MODEL Ch, I & MODEL Ch, II \\
\hline option A & $6^{*}$ & $5.24^{*}$ & 3.68 & $3.865^{*}$ \\
\hline option B & 4 & 4 & $3.745^{*}$ & 3.745 \\
\hline
\end{tabular}

Remark 4.5 If there is no other facility (e.g. hospital) near $Y_{1}\left(L_{1}-L_{6}\right.$ layout), as illustrated in the previous example, the coverage degree of the location $L_{1}$ where is located $Y_{1}$ corresponds to $\mu\left(\left\{Y_{1}\right\}\right)$, more precisely, it corresponds to the quality of $Y_{1}$. On the other hand, if the layout $L_{2}-L_{5}$ is observed, hospitals are close, thus the coverage of the location $L_{2}$ corresponds to $\mu\left(\left\{Y_{2}, Y_{5}\right\}\right)$, i.e., to the joint measure of facilities $Y_{2}$ and $Y_{5}$. 


\section{Conclusion}

This paper presents a generalization of the MCLP obtained by the incorporation of the Choquet integral into FMCLP. The nature of the observed integral takes into consideration the joint influence of each facility combination, which has not been done in any type of location problem before. The introduction of fuzzy integrals into the FMLCP makes the model more flexible and adaptable to real life problems. As it can be seen from (4), expert opinion of a Decision Maker given through set-function $\mu$ has a direct influence on the result. Thus a practical need for a new type of location problem is justified, and will further be called Extended FMCLP.

\section{Acknowledgement}

The authors would like to thank Humberto Bustince for providing the idea to use fuzzy integrals in FMCLP. This was suggested during a discussion at the FSTA 2014 conference.

This work was supported by the Ministry of Science and Technological Development of Republic of Serbia.

\section{References}

[1] V. Batanovic, D. Petrovic, R. Petrovic: Fuzzy Logic-based Algorithms for Maximum Covering Location Problems, Information Sciences 179 (1-2) (2009) 120-129, DOI: 10.1016/j.ins.2008.08.019

[2] P. Benvenuti, and R. Mesiar: "Integrals with Respect to a General Fuzzy Measure", Fuzzy Measures and Integrals (M. Grabisch, T. Murofushi, M. Sugeno eds.) Physica-Verlag (Springer-Verlag Company), Heidelberg 2000, 205-232

[3] P. Benvenuti, R. Mesiar, D. Vivina: "Monotone Set Functions-based Integrals", Handbook of Measure Theory (E. Pap ed.), Elsevier, Amsterdam, 2002, 1329-1379

[4] G. Choquet, "Theory of Capacities": Annales de l'Institut Fourier 5 (1953), $131-295$

[5] R. Church and C. ReVelle: Maximal Covering Location Problem, Papers of the Regional Science Association 32 (1974) 101-118

[6] R. Church: The Planar Maximal Covering Location Problem, Journal of Regional Science 2(24) (1984) 185-201

[7] J. R. Current and J. E. Storbeck: Capacitated Covering Models, Environment and Planning B: Planning and Design 15(2) (1988) 153-163

[8] J. Darzentas: A Discrete Location Model with Fuzzy Accessibility Measures, Fuzzy Sets and Systems 23 (1987) 149-154, DOI: 10.1016/01650114(87)90106-0 
[9] S. Davari, M. H. F. Zarandi, A. Hemmati: Maximal Covering Location Problem (MCLP) with Fuzzy Travel Times, Expert Systems with Applications $38 \quad$ (12) (2011) 14535-14541, DOI: 10.1016/j.eswa.2011.05.031

[10] D. Deneberg: Non-Additive Measure and Integral, Kluwer Academic Publishers, Dordrecht-Boston-London, 1994

[11] M. Detyniecki: Fundamentals on Aggregation operators,

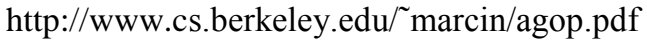

[12] R. Z. Farahani, N. Asgari, N. Heidari, M. Hosseininia, M. Goh: Covering Problems in Facility Location: A Review, Computers and Industrial Engineering 62 (2012) 368-407, DOI:10.1016/j.cie.2011.08.020

[13] M. Grabisch: k-additive Fuzzy Measures, $6^{\text {th }}$ International Conference on Information Processing and Management of Uncertainty in KnowledgeBased Systems (IPMU), Granada, Spain, July 1996

[14] M. Grabisch, J. Marichal, R. Mesiar, and E. Pap: Aggregations Functions, Cambridge University Press, 2009

[15] M. Grabisch, H.T. Nguyen, and E.A. Walker: Fundamentals of Uncertainty Calculi with Applications to Fuzzy Inference, Kluwer Academics Publishers, Dordrecht, 1995

[16] E. P. Klement, R. Mesiar and E. Pap: Triangular Norms, Series: Trends in Logic, Kluwer Academic Publishers, Vol. 8, Dordrecht 2000

[17] A. T. Murray, D. Tong, and K. Kim: Enhancing Classic Coverage Location Models, International Regional Science Review 33(2) (2010) 115-133, DOI: $10.1177 / 0160017609340149$

[18] E. Pap: Null-Additive Set Functions, Kluwer Academic Publishers, Dordrecht, 1995

[19] J. A. M. Perez, J. M. M. Vega, J. L. Verdegay: Fuzzy Location Problems on Networks, Fuzzy Sets and Systems 142 (2004) 393-405, DOI: 10.1016/S0165- 0114(03)00091-5

[20] C. ReVelle, and K. Hogan: The Maximum Availability Location Problem, Transportation Science 23 (1989) 192-200

[21] A. Takaci, M. Maric, D. Drakulic: The Role of Fuzzy Sets in Improving Maximal Covering Location Problem (MCLP). Procc. of SISY 2012, Subotica, Serbia 103-106

[22] Z. Wang, and G. J. Klir: Generalized Measure Theory, Springer, 2000

[23] R. R. Yager: "On Ordered Weighted Averaging Aggregation Operators in Multicriteria Decision Making," IEEE Transactions on Systems, Man and Cybernetics 18(1988), 183-190 